\title{
Effect of UV Radiation Applied Fractionally or Continuously on Stability of Biosensor Receptor Layer Component
}

\author{
A. KŁos-Witkowska* And K. Kajstura \\ Faculty of Mechanical Engineering and Computer Science, \\ Department of Computer Science and Automatics, University of Bielsko-Biala, \\ Willowa 2, 43-309 Bielsko-Biala, Poland
}

Received: 07.04.2020 \& Accepted: 15.09.2020

Doi: 10.12693/APhysPolA.138.781

*e-mail: awitkowska@ath.bielsko.pl

\begin{abstract}
This work includes an examination report of the influence of external conditions on the stability of the receptor layer cross-linking component in biosensors. Observations and three types of analysis resulting from the conducted research are presented. The studies described the influence of UV radiation (fractional: $1 \times 5 \mathrm{~min}, 2 \times 5 \mathrm{~min}, 3 \times 5 \mathrm{~min}$ and continuous $5 \mathrm{~min}, 10 \mathrm{~min}, 15 \mathrm{~min}$ ) exposure. A new type of analysis was carried out to compare the impact of external factors affecting the stability of the receptor layer cross-linking component in biosensors. An approach of numerical interpretation of the qualitative changes of stability has been offered. The tested factors influence the stability of the tested substance. All applied factors reduce the stability of the biosensors receptor layer component, however the dynamics of changes occurring under the influence of various types of UV application are different. It has been observed and confirmed by analyses that the effects caused by the external factor depend on the way of application (continuous or fractional exposure) and time of irradiation.
\end{abstract}

topics: biosensor, BSA, UV/Vis, receptor layer

\section{Introduction}

In the light of the COVID-19 pandemic, quick and reliable diagnostics are becoming even more important. Although the maturity to invent the SARS-CoV-2 virus biosensor is still far away, perhaps - thanks to the efforts of scientists it will be possible to respond to this challenge in the future. According to the definition given by the International Union of Pure and Applied Chemistry (IUPAC 1999), a biosensor is a selfcontained, integrated device which is capable of proving specific quantities or semi-quantities analytical information using a biological recognition element kept in direct spatial contact with an electrochemical transduction element [1]. The development of biosensors is important for many reasons including the need to monitor environmental cleanliness [2-4].

In the area of science, topics related to biosensors are developing dynamically and they can be split as follows:

- development of biosensors which could detect more than one analyte $[5,6]$,

- miniaturization of the existing biosensors $[7,8]$,

- creation of mathematical models and development of new models [9, 10],

- application of innovative materials (e.g., graphene, DNA) in biosensors [11, 12],
- improvement of biodetection by using nanotechnology achievements [13, 14],

- creation of new theoretical analyses which help track the current state of knowledge, presenting the issue of biosensors from a different perspective $[15,16]$,

- improvement of the current stage sensors by enhancing their sensitivity, selectivity, repeatability and reliability of measurements [17-19].

Among them, one can also distinguish tests of improvement of biosensor parameter stability. New results in this area constitute a contribution to the development of this field of science. Amidst the parameters influencing biosensors' operation, the issue of stability can be singled out. This problem is important as the stability of parameters of a device translates into its feasible operational lifetime.

Stability in biosensors is often addressed by research groups. The problem is open and will undoubtedly remain open in the future. In line with current scientific trends, the presented work examined the impact of UV radiation applied fractionally and once on the stability of the biosensor receptor layer component. This component was bovine serum albumin (BSA). Among the examined methods of immobilization, the cross-linking with BSA is superior to other methods in terms of sensitivity, detection threshold, reaction time as well as 
operational and thermal stability [20]. The biosensor receptor layer cross-linking component used in the examinations is often applied when designing new immunochemical tests [21]. It also defines the standard of proteins on a glass bed coated with surfactant-modified (ITO) electrodes [22].

The tested external factor affecting BSA stability was UV radiation. It is known from the literature that this radiation has a destructive effect on the protein. Research of Chinnathambi [23] was devoted to the effect of moderate UV-C irradiation on BSA and the complex with antimetabolite 5-fluorouracil: fluorescence spectroscopic and molecular modeling, Michnik [24] described the effect of UVC radiation on a conformational restructuring of human serum albumin and Zuorro [25] took into consideration the protection of human albumin against UV-C irradiation by natural antioxidants.

Nevertheless, there is no answer in the literature as to whether the same effect is obtained both with the fractional and the continuous use of an external factor. Does the method of application affect the dynamics of changes in the protein? Three types of analysis were applied to answer these questions. The presented work complements the existing knowledge and contributes to the development of science.

\section{Material and methods}

Aqueous BSA solutions with a concentration of $2 \mathrm{mg} / \mathrm{ml}$ were tested. Research material (bovine serum albumin) in the form of a solid, crystallized and lyophilized powder (99\% purity, lot: SLBK3063V) was purchased from Sigma Aldrich. The choice of BSA $2 \mathrm{mg} / \mathrm{ml}$ was a consequence of earlier studies [19]. Tests were performed on aqueous solutions on the basis of scientific reports [24] that such a solution provides a better understanding of the physicochemical properties of the test substances. The solutions were exposed to an electromagnetic field with a wavelength $\lambda=395 \mathrm{~nm}$ : fractionally $1 \times 5 \mathrm{~min}, 2 \times 5 \mathrm{~min}, 3 \times 5 \mathrm{~min}$ and once during $5 \mathrm{~min}, 10 \mathrm{~min}$ and $15 \mathrm{~min}$ on the first day of the experiment. The solutions were exposed to radiation in a $10 \times 10 \times 30 \mathrm{~mm}^{3}$ quartz cuvette. The cuvette was placed directly in front of the sources while the illumination was carried out in a darkened system isolated from external light.

Both for the samples radiated once $(1 \times 5 \mathrm{~min})$ and continuously (5 $\mathrm{min})$, a $5 \mathrm{~min}$ radiation is the reference point for radiation $(2 \times 5 \mathrm{~min})$, where after a $5 \mathrm{~min}$ radiation there is a pause and a $5 \mathrm{~min}$ radiation occurs again, and for radiation applied continuously for $10 \mathrm{~min}$.

It looks similarly in the case of $3 \times 5 \mathrm{~min}$. Radiation of $5 \mathrm{~min}$, pause, radiation of $5 \mathrm{~min}$, pause, radiation of $5 \mathrm{~min}$ and for radiation applied continuously, $15 \mathrm{~min}$. With the use of dual-beam $\mathrm{UV} / \mathrm{V}$ is spectrophotometer (Hallo DB-20 R) from
Dynamica, the absorption spectra of aqueous BSA solutions in the range of $220-350 \mathrm{~nm}$ were collected during days $1,2,4,5,6,7,8,11,13,14$ and 15 of the experiment.

The spectrum range was selected in such a way that it was possible to track changes in the absorption peak of $277 \mathrm{~nm}$, originating from aromatic resistors present in the tested protein, such as tryptophan (Trp), tyrosine (Tyr) and phenylalanine (Phe) [26]. The tests were carried out in three series (three times in each series) of measurements to avoid accidental errors and confirm the obtained effect.

\section{Results}

Figure 1 shows the spectra of the BSA aqueous solution on the 1st and 14th day of the experiment, in the range of $250-350 \mathrm{~nm}$ obtained by $\mathrm{UV} / \mathrm{V}$ is spectroscopy. The observed absorption peak appearing in $277 \mathrm{~nm}$ is derived from aromatic amino acids found in the tested components, such as tyrosine (Tyr), phenylalanine (Phe) and tryptophan (Thr). An increase in the absorption value with time has been observed. Losing stability by the biosensor receptor layers component could be tracked by changing (increasing) the value of the absorption coefficient. It reflects the rearrangement in the local environment of the ordered polypeptide chain and changes in the secondary structure of the protein. The changes identify an increase in the amount of helix conformation and decrease beta-sheet structure which leads to the loosening of the protein skeleton [27, 28]. The marked differences in the $300-340 \mathrm{~nm}$ range indicate the formation of protein aggregates, following protein denaturation. These results indicate that the aromatic residue environment has induced some changes for a prolonged time $[21,23]$.

The maximum of the broad peak at $277 \mathrm{~nm}$ and the local minimum at the wavelength range of $251 \mathrm{~nm}$ were used to analyze the stability of the biosensor receptor layer component. For this purpose, a model for changes in protein conformation proposed by Michnik [24] was used. According

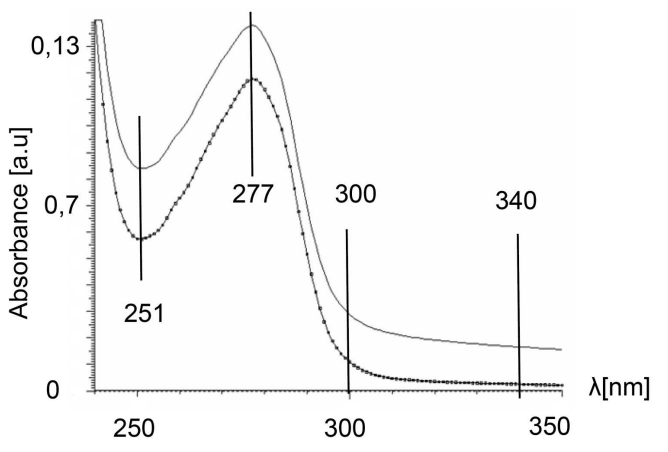

Fig. 1. UV/Vis spectra of BSA $(2 \mathrm{mg} / \mathrm{ml})$ solution without exposure to UV radiation on the 1st day (pointed line) and the 14th day (smooth line). 

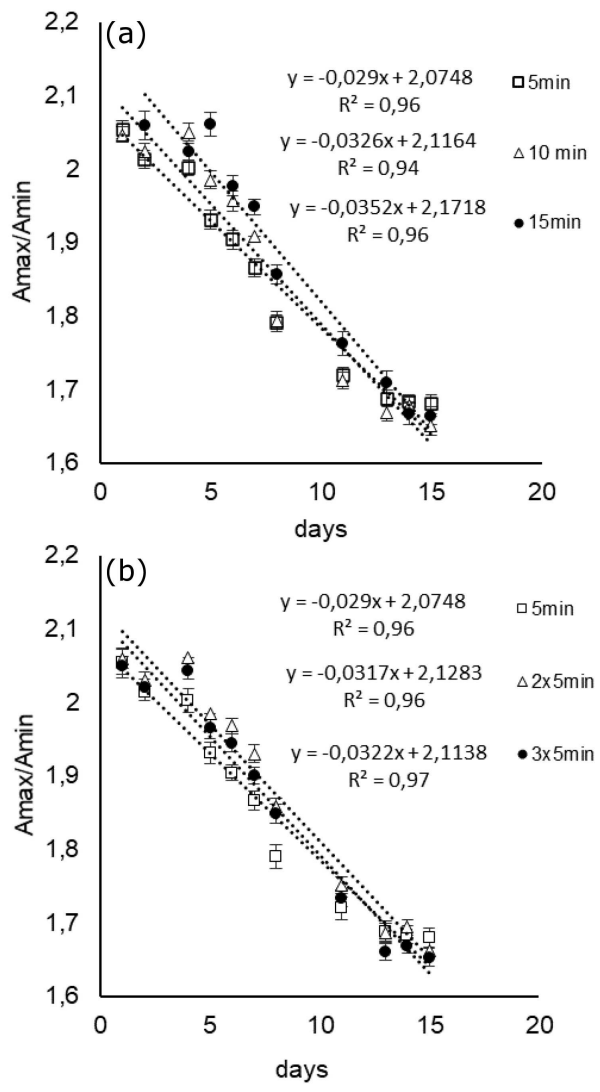

Fig. 2. Changes of the parameter $\left(A_{\max } / A_{\min }\right)$ on the following days of the experiments (a) for continuous irradiation: $5 \mathrm{~min}, 10 \mathrm{~min}, 15 \mathrm{~min}$, (b) for fractional irradiation: $5 \mathrm{~min}, 2 \times 5 \mathrm{~min}, 3 \times 5 \mathrm{~min}$.

to the assumptions of the model, the $A_{\max } / A_{\text {min }}$ parameter was calculated. The ratio of absorbances at the local maximum $A_{\max }$ at $k=277 \mathrm{~nm}$ and the local minimum $A_{\min }$ at $k=251 \mathrm{~nm}$ for the BSA spectra in the range of $250-280 \mathrm{~nm}$ has been chosen based on research of Artyukhov et al. [29]. According to them, $A_{\max } / A_{\min }$ can be considered as an indicator of structural changes in the bovine serum albumin molecules. They suggested that a decrease of this ratio was associated with destructive processes in the aromatic amino acids of the protein [29].

A similar analysis has been performed for the presented report to study the effect of UV radiation applied fractionally and once on the stability of the biosensor receptor layer component. The results are shown in Fig. 2.

Figure $2 \mathrm{a}$ shows changes of the parameter $A_{\max } / A_{\min }$ on the following days of the experiments. The decrease of the $A_{\max } / A_{\min }$ parameter with time, according to the model assumptions, suggests destructive processes in the aromatic amino acids of the protein. This process occurs in 5, 10, and $15 \mathrm{~min}$ radiated samples. The speed of changes is slightly different, as evidenced by the value of the parameter of an adjusted linear trend $y=a x-b$. The parameter indicates the dynamics of changes taking place. For 15 min radiated samples, the value of parameter $a$ is the largest
TABLE I

Comparison of changes rate for samples exposed to UV radiation applied fractionally and continuously.

\begin{tabular}{c|c|c}
\hline \hline Radiation & $a$ & $R^{2}$ \\
\hline $3 \times 5 \mathrm{~min}$ & $-0.0322 \pm 0.0008$ & $97(0.943)$ \\
$15 \mathrm{~min}$ & $-0.0352 \pm 0.0012$ & $96(0.950)$ \\
$2 \times 5 \mathrm{~min}$ & $-0.0317 \pm 0.0011$ & $96(0.956)$ \\
$10 \mathrm{~min}$ & $-0.0326 \pm 0.0009$ & $94(0.952)$
\end{tabular}

and it is equal to $-0.0352 \pm 0.0012$, with $R^{2}=96$. For 10 min radiated samples, $a$ is slightly smaller, namely $a=-0.0326 \pm 0.0009$ and $R^{2}=94$, and for 5 min irradiated samples, $a=-0.029 \pm 0.001$ and $R^{2}=96$.

It can be observed that the fastest destructive change in the aromatic amino acids of the protein occurs in samples radiated the longest, i.e., $15 \mathrm{~min}$, followed by $10 \mathrm{~min}$ and $5 \mathrm{~min}$. Therefore, one can conclude that the longer the exposure to UV radiation, the faster the destructive changes in the protein occur. Note also that the prolonged exposure to UV radiation destabilizes the cross-linking component of the biosensor receptor layer and, further, is proportional to the time of exposure. A similar analysis has been performed for samples exposed fractionally to UV radiation during $5 \mathrm{~min}$, $2 \times 5 \mathrm{~min}, 3 \times 5 \mathrm{~min}$ (Fig. $2 \mathrm{~b}$ ). The value obtained for $1 \times 5$ min is: $a=-0.029 \pm 0.001, R^{2}=96$, for $2 \times 5 \mathrm{~min}$ is: $a=-0.0317 \pm 0.0011, R^{2}=96$ and for $3 \times 5 \mathrm{~min}$ is: $a=-0.0322 \pm 0.0008, R^{2}=97$. It can be seen that the fastest changes are taking place for samples radiated the highest number of times $(3 \times 5 \mathrm{~min})$ and then for $(2 \times 5 \mathrm{~min})$ radiated samples and finally for $(1 \times 5 \mathrm{~min})$ radiated samples. The changes in $A_{\max } / A_{\min }$ ratio under UV radiation may be connected with the conformational restructuring of the albumin molecule, including a reorganization of the regions where aromatic amino acids (tryptophan and tyrosine) are situated. The loss of stability due to UV radiation applied fractionally to the cross-linking component of the biosensor receptor layer is directly proportional to the applied radiation fraction.

The rate of changes occurring in the protein under the influence of UV radiation administered continuously and fractionally is shown in Table I. Comparing these results of parameter $a$, it can be seen that the dynamics of changes are slightly greater for samples with continuous exposure than for samples subjected to fractional exposure.

A similar effect was obtained by performing a different type of analysis (see Fig. 3). Relative changes in absorption occurring in the cross-linking component of the biosensor receptor layer over time were determined. The values are given in percent. Changes were analyzed relative to the value of the absorption parameter on the first day of the experiment $\left(A_{1}\right)$. Index $n$ indicates the time of radiation and the way of exposure to an external factor. 
Relative changes in absorption over time due to electromagnetic field exposition during 5 min, $10 \mathrm{~min}, 15 \mathrm{~min}, 2 \times 5 \mathrm{~min}, 3 \times 5 \mathrm{~min}$.

\begin{tabular}{c|c|c|c|c|c|c}
\hline \hline \multirow{2}{*}{ Days } & \multicolumn{5}{|c}{$A_{n} / A_{1} \times 100 \%$} \\
\cline { 2 - 6 } & Ref. & $5 \mathrm{~min}$ & $10 \mathrm{~min}$ & $15 \mathrm{~min}$ & $2 \times 5 \mathrm{~min}$ & $3 \times 5 \mathrm{~min}$ \\
\hline 1 & $0 \pm 0.0$ & $0 \pm 0.2$ & $0 \pm 0.1$ & $0 \pm 0.2$ & $0 \pm 0.3$ & $1 \pm 0.2$ \\
2 & $1 \pm 0.2$ & $1 \pm 0.4$ & $1 \pm 0.1$ & $1 \pm 0.2$ & $1 \pm 0.1$ & $2 \pm 0.1$ \\
4 & $0 \pm 0.1$ & $2 \pm 0.5$ & $1 \pm 0.2$ & $0 \pm 0.1$ & $0 \pm 0.0$ & $1 \pm 0.1$ \\
5 & $1 \pm 0.4$ & $3 \pm 0.1$ & $2 \pm 0.2$ & $2 \pm 0.3$ & $1 \pm 0.0$ & $3 \pm 0.4$ \\
6 & $2 \pm 0.3$ & $3 \pm 0.1$ & $3 \pm 0.3$ & $2 \pm 0.3$ & $1 \pm 0.1$ & $3 \pm 0.2$ \\
7 & $4 \pm 0.0$ & $6 \pm 0.3$ & $5 \pm 0$ & $7 \pm 0.2$ & $4 \pm 0.2$ & $5 \pm 0.1$ \\
8 & $5 \pm 0.3$ & $8 \pm 0.3$ & $9 \pm 0.2$ & $10 \pm 0.1$ & $6 \pm 0.1$ & $7 \pm 0.3$ \\
11 & $11 \pm 0.2$ & $10 \pm 0.5$ & $12 \pm 0.1$ & $10 \pm 0.1$ & $9 \pm 0.2$ & $11 \pm 0.1$ \\
13 & $14 \pm 0.1$ & $12 \pm 0.4$ & $14 \pm 0.1$ & $13 \pm 0.1$ & $11 \pm 0.2$ & $14 \pm 0.1$ \\
14 & $17 \pm 0.1$ & $13 \pm 0.2$ & $15 \pm 0.2$ & $14 \pm 0.0$ & $12 \pm 0.1$ & $15 \pm 0.1$ \\
15 & $17 \pm 0.2$ & $13 \pm 0.3$ & $17 \pm 0.3$ & $18 \pm 0.3$ & $15 \pm 0.1$ & $16 \pm 0.2$
\end{tabular}
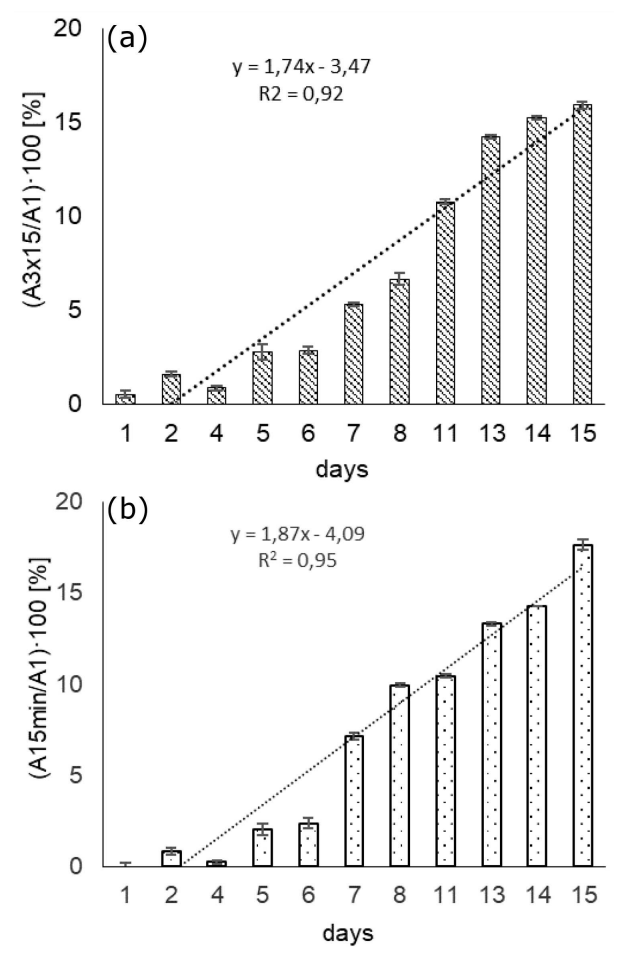

Fig. 3. Relative changes in absorption during 15 days for fractionally radiated samples $(3 \times 5 \mathrm{~min})$ and continuously radiated samples (15 min).

The percentage increase in the absorption coefficient is noticeable with time. The maximum value is visible on the 15 th day of the experiment and for the fractionally radiated samples $(3 \times 5 \mathrm{~min})$ it is $16 \pm 0.2 \%$ (see Fig. 3a), while for the samples exposed to continuous UV radiation (15 min) it is $18 \pm 0.3 \%$ (see Fig. $3 \mathrm{~b}$ ). The remaining percentages for samples subjected to continuous and fractional exposition are presented in Table II.

The behaviour in the fit function $y=a x+$ $b$ of $a$ parameter clearly indicates that for samples radiated continuously (15 $\mathrm{min})$ this parameter has a higher value than for samples subjected to fractional exposure $(3 \times 5 \mathrm{~min})$. Therefore, the compliance occurs with earlier performed analyses concluding that changes in the stability of the receptor layer component occur more rapidly for continuously radiated samples than for fractionally radiated samples.

It can therefore be concluded that the denaturation of the tested cross-linking component of biosensor receptor layers occurs faster after the application of the agent in a continuous form than after the application of the agent fractionally.

According to [30], the result of the loss of stability is associated with protein denaturation. A change in the distance in the polypeptide chain results in a change in the strength of the interaction between amino acids. The tertiary structure of the biosensor receptor layer component is disturbed which results in the loss of biological activity of the protein, occurring faster under the influence of a continuous rather than fractional exposure.

Another type of analysis has been performed to determine which of the applied factors most affected the stability of the receptor layer cross-linking component in biosensors. For this purpose, relative percentage differences were calculated based on

$$
K=\left(\frac{A_{1}}{A_{0}}\right) \times 100 \%,
$$

where $A_{1}$ is the absorption under the influence of the factor in the following days of the study and $A_{0}$ is the absorption of reference samples (without radiation) based on

$$
M=100-K \text {. }
$$

The percentages of changes in individual days $M_{1}$, $M_{2}, M_{3}, \ldots, M_{y}$ were also calculated. When adding up their values, the relation on $M_{D x}$ is obtained, where $x=1,2, \ldots$,

$$
\begin{aligned}
& M_{1}+M_{2}=M_{D 1}, \\
& M_{2}+M_{3}=M_{D 2}, \\
& M_{3}+M_{4}=M_{D 3} .
\end{aligned}
$$




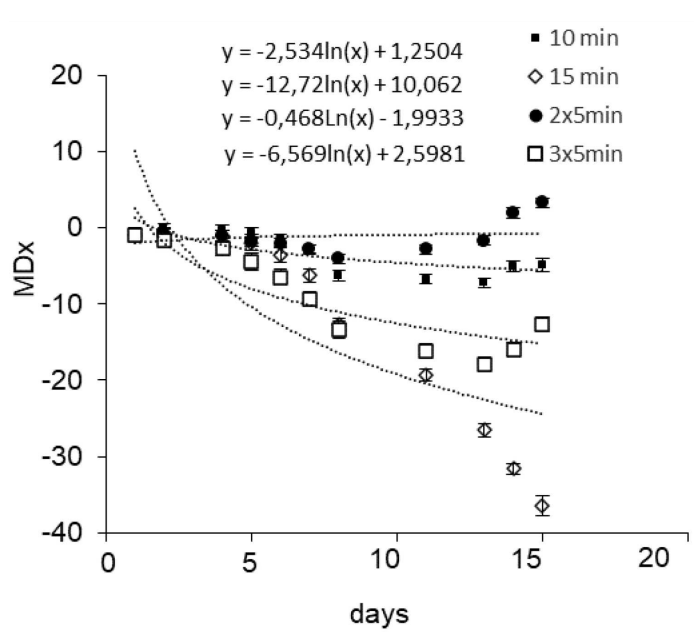

Fig. 4. $M_{D x}$ matching with the description of logarithmic functions for UV exposition: $10 \mathrm{~min}$, $15 \mathrm{~min}, 2 \times 5 \mathrm{~min}, 3 \times 5 \mathrm{~min}$.

A visual illustration of the change in $M_{D x}$ value in subsequent days of the experiment was made and the fitting functions of the logarithmic trend have been performed to the results. The $M_{D x}$ matching with the description of logarithmic functions in the form $y=a \ln (x) \pm b$ for selected coefficients is presented in Fig. 4. Note that a sloping character of $y_{1}, y_{2}, y_{3}, y_{4}$ can be observed. One can interpret it in such a way that the parameter $a$ in the description of the logarithmic function $y=a \ln (x)+b$ is a parameter demonstrating the nature of changes in stability. When a decrease in stability of the biosensor receptor layer component is noticeable, then this parameter takes a negative value.

Comparing the coefficients $a$ with the logarithmic function allows to deduce the following: (i) a comparison of 15 min with $3 \times 5$ min gives a higher factor at $15 \mathrm{~min}$, i.e., $a=-12.721$, than at $3 \times$ 5 min, i.e., $a=-6.5693$. This means that samples radiated continuously change faster when subjected to fractional exposure; (ii) a comparison of $10 \mathrm{~min}$ with $2 \times 5 \mathrm{~min}$ gives a higher factor at $10 \mathrm{~min}$, i.e., $a=-2.5339$, than at $2 \times 5 \mathrm{~min}$, i.e., $a=-0.468$. This means that samples radiated continuously change faster when subjected to fractional exposure.

The weakening of protein stability is also affected by the solvent which - in the conducted studies is water. Denaturation of globular proteins is usually ascribed to hydrophobic hydration of polar surfaces. Increasing the time of UV exposition may be associated with diminishing the fraction of buried non-polar side-chains contact water on unfolding. It can be supposed that the differences observed in parameter changes between the continuously radiated sample and the fractionally radiated sample are connected with water reorganization in albumin macromolecule changed by UVC radiation.

It is observed that all curves are below zero, thus it can be concluded that UV radiation adversely affects the protein accelerating its aging.

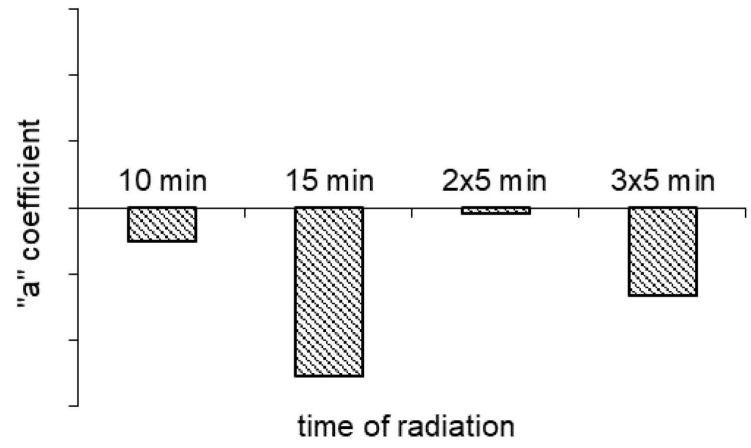

Fig. 5. Factors reducing the stability of the crosslinking component of biosensor receptor layers.

In the third analysis presented, compliance with earlier analyses was obtained, confirming the effect of faster destabilization of the biosensor receptor layer component under the influence of radiation applied continuously than radiation applied in a fractional way.

Comparing $a$ coefficients of the logarithmic function in Fig. 5, one can notice a higher factor at $15 \mathrm{~min}(-12.721 \pm 0.9)$ than at $3 \times 5 \mathrm{~min}$ $(-6.5693 \pm 0.4)$. This means that samples radiated continuously change faster then samples exposed to fractional radiation. The similar effect can be seen during a comparison of $10 \mathrm{~min}(-2.5339 \pm 0.02)$ with $2 \times 5 \min (-0.468 \pm 0.09)$.

\section{Conclusions}

An approach of numerical interpretation of the qualitative changes of stability under the influence of UV radiation (fractional: $1 \times 5 \mathrm{~min}$, $2 \times 5 \mathrm{~min}, 3 \times 5 \mathrm{~min}$ and continuous: $5 \mathrm{~min}, 10 \mathrm{~min}$, $15 \mathrm{~min}$ ) exposure is offered in this paper.

It allows to sum up the test results related to the influence of external conditions on the stability of the cross-linking component of biosensor receptor layers and it can be stated that the tested factors influence the stability of the tested substance. All applied factors reduce the stability of the biosensors receptor layer component, however, the dynamics of changes occurring under the influence of various types of UV applications are different. It has been observed and confirmed by analyses that the effects caused by external factors depend on the application way (continuous or fractional exposure) and the time of irradiation.

A greater impact was observed for continuously radiated samples (15 min) than for samples subjected to $(3 \times 5 \mathrm{~min})$ fractional exposure. Similarly, for $10 \mathrm{~min}$ - a more pronounced effect than for $2 \times 5$ min was noted. This is because the conformational changes of the biosensor receptor layer component occur more rapidly when exposed in a continuous way than when exposed in a fractional way. The stability changes also depend on the time of exposure and are more visible for $15 \mathrm{~min}$ than for $10 \mathrm{~min}$ and $5 \mathrm{~min}$. 
The observed effects can be connected to:

- rearrangement in the local environment of the ordered polypeptide chain,

- changes in the secondary structure of the protein,

- increase in the amount of helix conformation and decrease of beta-sheet structure (which leads to the loosening of the protein skeleton),

- formation of protein aggregates leading to protein denaturation,

- destructive processes in the aromatic amino acids of the protein,

- distance change in the polypeptide chain,

- weakening of protein stability affected by the solvent,

- water reorganization in the albumin macromolecule changed by UV radiation.

\section{References}

[1] D. Thévenot, K. Toth, R. Durst, G. Wilson, Biosens. Bioelectron. 16, 121 (2001).

[2] O. Mitryasova, V. Pohrebennyk, M. Cygnar, I. Sopilnyak, in: Proc. 16th Int. Multidisciplinary Scientific GeoConference Surveying Geology and Mining Ecology Management, SGEM 2016, p. 317.

[3] V. Pohrebennyk, A. Petryk, in: 17th Int. Multidisciplinary Scientific GeoConference Surveying Geology and Mining Ecology Management, SGEM 2017, p. 967.

[4] S. Girotti, E. Nora Ferri, M.G. Fumo, E. Maiolini, Analyt. Chim. Acta 608, 901 (2008).

[5] G. Tuerdean, Int. J. Electrochem. 2001 , 343125 (2011).

[6] J. Pilas, Y. Yazici, T. Selmer, M. Keusgen, M. Schöning, Sensors 18, 1470 (2018).

[7] B. Derkus, Biosens. Bioelectron. 79, 901 (2016).

[8] J. Kim, A. Campbell, B.E.F. de Ávila, J. Wang, Nature Biotechnol. 37, (2019).

[9] T. Rajba, A. Kłos-Witkowska, S. Ivasiev, I. Yakymenko, M. Kasianchuk, in: Proc. IEEE 9th Int. Conf. on Intelligent Data Acquisition and Advanced Computing Systems: Technology and Applications, IDAACS'201\%, Bucharest 201710.1109/IDAACS.2017.8095054, IEEE, 2017, p. 82

[10] V. Martsenyuk, K. Warwas, K. Augustynek, A. Kłos-Witkowska, V. Karpinskyi, N. Klymuk, Z. Mayhruk, in: Proc. 16th Int. Conf. on Control, Automation and Systems, Gyeongju (South Korea), 2016, IEEE, 2016, p. 489.
[11] T. Kuila, S. Bose, P. Khanra, A.K. Mishra, N. HoonKim, J. Hee, Biosens. Bioelectron. 26, 4637 (2011).

[12] A. Al-Ghamdi, O. Al-Hartomy, R. Gupta, F. El-Tantawy, E. Taskan, H. Hasar, F. Yakuphanoglu, Acta Phys. Pol. A 121, 673 (2012).

[13] L. Mosińska, P. Popielarski, K. Fabisiak, A. Dychalska, Opt. Mater. 101, 109676 (2020).

[14] I. Ennen, C. Albon, A. Weddemann et al., Acta Phys. Pol. A 121, 420 (2012).

[15] A. Kłos-Witkowska, V. Martsenyuk, V. Karpinskyi, Acta Phys. Pol. A $\mathbf{1 3 5}$, 375 (2019).

[16] V. Martsenyuk, A. Kłos-Witkowska, A. Sverstiuk, Electron. J. Qual. Theory Differ. Equ. 27, 1 (2018).

[17] F. Ricci, A. Amine, G. Palleschi, D. Moscone, Biosens. Bioelectron. 18, 165 (2003).

[18] E. Songa, J. Okonkwo, Talanta 155, 289 (2016).

[19] A. Kłos-Witkowska, Acta Phys. Pol. A 133, 101 (2018).

[20] C. Sarika, K. Rekha, B. Narasimha Murthy, 3 Biotech 5, 911 (2015).

[21] G. Ertürk, D. Berillo, M. Hedström, B. Mattiasson, Biotechnol. Rep. (Amst) 3, 65 (2014).

[22] Ch.H. Lin, M.J. Lee, W. Lee, Appl. Phys. Lett. 109, 093703 (2016).

[23] S. Chinnathambi, S. Karthikeyan, D. Velmurugan, N. Hanagata, P. Aruna, S. Ganesan, Int. J. Spectrosc. 2015, 315764 (2015).

[24] A. Michnik, K. Michalik, Z. Drzazga, J. Therm. Anal. Calorim. 80, 399 (2005).

[25] A. Zuorro, R. Lavecchia, Am. J. Biochem. Biotechnol. 14, 247 (2018).

[26] Hongliang Xu, Nannan Yao, Haoran Xu, Tianshi Wang, Guiying Li, Zhengqiang Li, Int. J. Mol. Sci. 14, 14185 (2013).

[27] Zhenxing Chi, Bowen Hong, Xiulian Ren, Kunyu Cheng, Yuqian Lu, Xinning Liu, Int. J. Rapid Commun. 51, 279 (2018).

[28] S. Servagent-Noinville, M. Revault, H. Quiquampoix, M. Baron, J. Coll. Interface Sci. 221, 273 (2000).

[29] V.G. Artyukhov, A.A. Pantyavin, G.A. Vashanov, J. Appl. Spectrosc. 68, 291 (2001).

[30] Youling L. Xiong, in: Quality in Frozen Food, Springer, Boston 1997, p. 111. 